\title{
The forecast and analysis of toll channel scale of expressway toll station
}

\author{
Guanzheng Pang ${ }^{1 *}$ \\ ${ }^{1}$ Shang Hai maritime university Transportation Engineering, Shang Hai China
}

\begin{abstract}
With the rapid growth of highway traffic, congestion of highway toll station has become a common phenomenon in peak hours. However, the congestion at the toll station has a serious impact on the normal operation of expressway. When the queuing vehicles overflow the toll station, the queue leader will lose control, and the operation of the main line vehicles will be seriously disturbed. This paper analyzes the causes of queuing phenomenon in toll stations, studies the characteristics of different toll channels and traffic flow, and then establishes the traffic flow theory model, and classifies the toll stations according to the average delay.
\end{abstract}

\section{Introduction}

Expressway is an indispensable hardware facility in a modern country, which is confirmed by the construction of Expressway in China. In order to make the highway play the role of economic artery, only through large-scale construction is not enough to achieve the goal. ${ }^{[1]}$ With the hardware infrastructure, but also equipped with a high level of management, in order to make full use of hardware facilities.

In the past ten years, China's car ownership is growing, and the existing road resources are relatively reduced. During peak hours, traffic congestion at highway toll stations has become a normal phenomenon. If this situation is not improved, the use effect of expressway will be greatly reduced. After a period of time, the utilization rate of expressway will decline.

The traffic congestion of Expressway main line toll station has become a traffic problem in various countries, which interferes with the operation of expressway. ${ }^{[2]}$ Therefore, the prediction and analysis of toll channel scale of toll station is directly related to the service level and operation efficiency of expressway. The research on toll station has an important and positive impact on Accelerating China's economic development and improving people's living standard Materials and Methods.

Based on the original design intention of compound passageway, the paper analyzes its capacity. ${ }^{[3]}$

The appropriate queuing model is selected to solve the system capacity of the toll station, and the capacity of all toll channels of the toll station is analyzed by the control variable method, and the applicability of the model is analyzed. According to the average delay time, the service level of toll station is divided reasonably, and it provides valuable reference for the determination of toll station channel number and location selection.

\section{Materials and Methods}

Compared with the road section, the traffic flow through toll station is more fragile. The failure of manual toll facilities and vehicles entering etc channel will cause the interruption of traffic flow. When vehicles pass through toll stations, they will pass through the following sections: upstream section, deceleration transition section, toll area section, acceleration transition section and downstream section.

Usually, when vehicles approach the toll area to pay, they need to enter the toll plaza at the deceleration transition section first. By observing the types of channels and the length of vehicle queue, different types of vehicles will enter the toll window with shorter queue length to trade after choosing the toll channel. Queuing payment process follows the principle of "first come first serve". After the completion of the payment service, the vehicle accelerates into the main lane or ramp in the transition section.

\section{1mtc channel layout design}

MTC channel is mainly composed of toll Island, toll lane and toll sentry box, which is generally set in parallel with the road axis to the shoulder side. The toll island is located between the toll lanes in the shape of a boat and is arranged in parallel with the center line of the road. ${ }^{[4]}$ Please refer to the table below for the width of toll lane and the size of toll island.

\footnotetext{
$\overline{{ }^{*} \text { Corresponding author: }{ }^{\mathrm{a}} 1242808052 @ q q . c o m}$
} 


\subsection{Analysis of toll lane scale determination}

\subsection{1 toll station traffic survey and data analysis}

In the process of vehicles passing through the toll station, in some peak hours with large traffic volume, due to the delay of toll collection, vehicle deceleration will produce queuing phenomenon. ${ }^{[5]}$ The delay of various vehicles in the queuing process is composed of the following three parts:

(1) the deceleration time of vehicles entering the toll station.

$$
t_{1}=\frac{v_{0}}{3.6 a_{1}}
$$

(2) Average length of stay of vehicles at toll stations:

$$
W=E(t)+T_{q}
$$

(3) The time for the vehicle to leave after receiving the service

$$
t_{2}=\frac{v_{0}}{3.6 a_{2}}
$$

Where, $v_{0}$ is the speed of normal traffic flow $(\mathrm{km} / \mathrm{h})$; $\mathrm{a} 1, \mathrm{a} 2$ is the deceleration and acceleration of the vehicle $\left(\mathrm{m} / \mathrm{s}^{2}\right)$; E (T) is the expected value of service time (s); Is the average queuing time of vehicles (s).

From the above analysis, we can get the total delay of vehicles passing through the toll station:

$$
d=\left(\frac{1}{\lambda}-\frac{3.6 m}{v_{0}}\right)\left\{\lambda E(t)+\frac{\lambda^{2}[E(t)]^{2}+\lambda^{2} D(t)}{2[1-\lambda E(t)]}\right\}+\frac{v_{0}}{7.2}\left(\frac{1}{a_{1}}+\frac{1}{a_{2}}\right)
$$

Where, $\lambda$ Is the average arrival rate of vehicles (PCU / $\mathrm{s}) ; \mathrm{M}$ is the conversion factor of fleet length $(\mathrm{m} / \mathrm{veh}) ; \mathrm{D}(\mathrm{t})$ is the variance of service time; The service traffic volume of the toll station can be obtained from the delay.

\subsection{2 calculation of capacity of toll lane in "double toll" mode}

The capacity of the compound channel depends on the number of toll booths. Due to the use of manual toll, the capacity of the channel is directly limited by the maximum service time in multiple toll windows. ${ }^{[6]}$

When the arrival of vehicles follows the Poisson process, the probability of $\mathrm{N}$ vehicles arriving in the toll channel in a certain time can be obtained by using the following formula.

$$
P_{n}(t)=\frac{(\lambda t)^{n}}{n !} e^{-\lambda t}
$$

Suppose the number of toll windows in the corridor is $\mathrm{n}$ and the service time of each vehicle is $\mathrm{X}_{\mathrm{r}}$. According to above equation, the distribution function can be obtained:

$$
F_{X_{1}}\left(X_{1}\right)=F_{X_{2}}\left(X_{2}\right)=F_{X_{n}}\left(X_{n}\right)=1-e^{-\mu x}
$$

In the duplex channel, if the rear vehicles in the queue finish the service before the front vehicles, they must wait for the front vehicles to finish the service before moving; Referring to the calculation method of solving the maximum distribution in probability theory, we can get the distribution function of the maximum value $\mathrm{m}$ of continuous service time:

$$
F_{M}(Z)=\prod_{r=1}^{n} F_{X_{r}}(z)=\left(1-e^{-\mu x}\right)^{n}=\sum_{r=0}^{n} c_{n}^{r}\left(-e^{-\mu x}\right)^{r}
$$

The probability density function is as follows:

$$
P(X)=F_{M}(Z)=\sum_{r=0}^{n}(-1) r \mu c_{n}^{r}\left(-e^{-\mu x}\right)^{r}
$$

According to the simultaneous formula, the capacity of two toll windows in series is 1.19 times of that of a single kiosk.

In the same way, we use the above method to solve the capacity of the multi toll channel with three, four and five toll windows. With the increase of the number of toll windows, the capacity increases gradually.

\subsection{3 analysis of etc passage capacity}

The minimum safe vehicle distance is the shortest driving distance when two adjacent etc vehicles meet the emergency stop of the vehicle in front, and the latter vehicle must take braking to avoid collision with the vehicle in front. The actual stopping distance is a dynamic change with the vehicle speed, which is specified in the code for design of highway route. Saturation headway $\mathrm{H}=\mathrm{L}_{\text {stop }} / \mathrm{v}$, we can get the capacity of etc toll channel

$$
C_{E}=\frac{1000}{L_{\text {stop }}} v
$$

\section{3 toll channel design}

The average queue length and average waiting time describe the service level of toll station from two dimensions of space and time, which are the most important indicators for evaluation. The service level of toll station can be classified according to the average delay and queue length of vehicles.

(1) Average queue length.

The queue length can be calculated according to the formula.

$$
L_{q}=\frac{\lambda D[T]+\lambda E^{2}[T]}{2 E[T](N-\lambda E(T))}\left[\sum_{i=}^{N-1} \frac{(N-1) !(N-\lambda E[T])}{i !(\lambda E[T])^{N-i}}\right]^{-1}
$$

Among them,

$\mathrm{L}_{\mathrm{q}}$ - average queue length, vehicle / Lane

$\lambda$ - Average incoming vehicle intensity, vehicle / $\mathrm{s}$,

$\mathrm{N}$-number of toll lanes

$E[T]$ - expected value of service time, s;

$\mathrm{D}[\mathrm{T}]$-variance of service time, $\mathrm{S}^{2}$

(2) Average delay time

When choosing the model to calculate the average vehicle delay of toll station, we should calculate the toll station as a whole, simply calculate the average delay of toll channel, the result will have error.

Similarly, when $\mathrm{m} / \mathrm{g} / \mathrm{K}$ queuing model is used to describe the traffic flow of toll station, the average delay time of vehicles can be calculated according to equation.

$$
d=\frac{D[T]+E^{2}[T]}{2 E[T](N-\lambda E(T))}\left[1+\sum_{i=0}^{N-1} \frac{(N-1) !(N-\lambda E(T))}{i !(\lambda E[T])^{N-i}}\right]^{-1}
$$




\subsection{1 analysis of toll station passage time}

Suppose that the traffic volume entering the toll station is $Q$, the flow rate is $V$, and the entry time is $T_{n}$. Time consuming of charging service section $\mathrm{T}_{\mu}$, Departure time $\mathrm{T}_{\omega}$. Therefore, the total consumption time is:

$$
T=T_{n}+T_{\mu}+T_{\omega}
$$

Where, $\mathrm{T}$ is the average of the total time consumed by vehicles passing through the toll station, $T_{\mu}$ is the average time spent in the toll service process, and the average of the time consumed by vehicles entering and leaving the transition period respectively.

\subsection{2 entering the transition section model:}

Because the expressway is completely closed, it is in line with the applicable conditions of hydrodynamics to regard the traffic flow upstream and downstream of toll station as continuous flow. Under this premise, assume that the upstream traffic flow of the toll station is $q_{0}$, and the flow rate is $v_{0}$. The process of vehicles entering the toll plaza is as follows

When the vehicle is located at the toll station $\mathrm{x}$, the traffic volume meets the following relationship:

$$
q_{0}=k(x) \cdot v(x)
$$

Where $\mathrm{v}(\mathrm{x})$ is the traffic flow velocity $(\mathrm{km} / \mathrm{h})$ at position $\mathrm{x}$, and $\mathrm{K}(\mathrm{x})$ is the traffic flow density at position $\mathrm{x},(\mathrm{pcu} / \mathrm{km})$.

The following formula can be obtained from the physical formula:

$$
T_{n}=\frac{n w_{n} a_{1}}{v_{0}\left(n d-w_{n}\right)} \operatorname{In}\left(\frac{n d}{w_{n}}\right)
$$

\subsubsection{Stage model of charging service:}

According to the queuing theory, the MTC channel queuing law follows the $\mathrm{M} / \mathrm{M} / 1$ system, then the toll station is a parallel system of multiple $\mathrm{M} / \mathrm{M} / 1$ subsystems. Use the average arrival rate obtained from the survey $\lambda$. And average service rate $\mu$, The average time consumed by vehicles in the queuing phase can be calculated

$$
T_{w}=\frac{1}{\mu-\lambda}
$$

\subsubsection{Leaving the paragraph model:}

This stage is similar to the first stage:

$$
T_{W}=-\frac{n^{2} d a_{1}}{v_{2}\left(n d-w_{u}\right)} \operatorname{In}\left(\frac{n d}{w_{u}}\right)
$$

According to the previous calculation, the total time of vehicles passing through the toll plaza is taken as the objective function:

$$
T=T_{n}+T_{u}+T_{W}=\frac{n w_{n} a_{1}}{v_{0}\left(n d-w_{n}\right)} \operatorname{In}\left(\frac{n d}{w_{n}}\right)+\frac{n}{n \mu-q_{1}}-\frac{n^{2} d a_{1}}{v_{2}\left(n d-w_{u}\right)} \operatorname{In}\left(\frac{n d}{w_{u}}\right)
$$

\section{Results \& Discussion}

At present, Gushan toll station adopts the traditional closed toll mode, and the channel layout adopts the traditional parallel mode. There are two types of toll channels: etc lane, MTC lane and TTC lane. The speed limit of etc lane is $20 \mathrm{~km} / \mathrm{h}$, and the minimum distance between vehicles is $9 \mathrm{~m}$. There are three toll channels at the entrance of the toll station. From left to right, there are one etc channel, one TTC channel and one MTC Lane (super wide lane). Etc lane is arranged near the central axis of the road, that is, the left side of the driving direction, and super wide lane is arranged on the right side of the driving direction. Five toll channels are set at the exit of the toll station. From left to right, there are one etc lane, two TTC lanes and one MTC Lane (super wide lane). The layout of etc channel and super wide lane is the same as that at the entrance. Usually, TTC lane is used as MTC lane. The width of inner lane is 3.2, and the width of super wide lane is $4 \mathrm{~m}$.

\subsection{TTC Channel service level prediction and analysis}

There are two TTC channels at the exit and one at the entrance of Gushan toll station, but all of them have not been put into use. Therefore, it is necessary to predict the capacity of TTC channel in order to calculate the service level of TTC channel.

When vehicles pass through the duplex toll channel, they will enter and leave the toll station together. The service time depends on the maximum service time of the two vehicles. As long as the vehicles in front or behind do not leave the toll station, the queuing vehicles can not enter the toll station to receive service. According to the references, we can use computer program to simulate the relationship between the number of toll windows and the length of stay. The simulation results are shown in the table below

Table 1 relationship between number of toll windows and length of stay

\begin{tabular}{ccccccc}
\hline Number of toll windows in toll channel & 1 & 2 & 3 & 4 & 5 & 6 \\
\hline Average vehicle stay time / S & 21.8 & 15.4 & 13.1 & 11.9 & 11.3 & 11.4 \\
Queue average service time / S & 21.8 & 30.8 & 39.3 & 47.6 & 56.5 & 68.4 \\
Tandem capacity / vehicle & 164 & 233 & 273 & 300 & 319 & 315 \\
Capacity increment & & 68 & 40 & 26 & 19 & -4 \\
Average service efficiency & 164 & 116 & 91 & 75 & 63 & 52 \\
\hline
\end{tabular}




\subsection{Determination of toll channel scale}

This paper uses the theoretical model of traffic flow based on travel time to determine the scale of toll channel. Assuming that the existing service time and traffic volume remain unchanged, the scale of toll channel is determined to minimize the travel time of each vehicle. After calculation, when the scale of toll channels at the entrance reaches 4 , the time for vehicles to pass through the toll station is the shortest.

\subsection{Design of rectification scheme}

Increasing the scale of toll channels is not necessarily feasible under the existing conditions. The effective way is to maximize the use of limited space resources under the existing conditions, standardize the management, shorten the service time to improve the service level. The transformation of single toll station into compound toll station means that the number of toll windows is increased longitudinally on the basis of single toll station to improve the service capacity, which can improve the traffic capacity of toll station under the constraint of limited space resources.

\section{Conclusion}

This paper comprehensively analyzes the process of different modes of toll passage and the behavior of vehicles passing through the toll station, and comprehensively studies the application of traffic flow theory in determining the scale of toll passage, mainly from the aspects of toll station design and toll passage selection. At present, $\mathrm{M} / \mathrm{G} / \mathrm{K}$ or $\mathrm{M} / \mathrm{G} / 1$ model is widely used in queuing model, but the arrival of vehicles may not conform to Poisson distribution when chi square test is carried out, so the model can not be used for calculation. In the future, although the application of multiple charging mode is less, it has great potential. Under normal circumstances, a duplex channel will be set with two charging windows. If more than two charging windows are set, what will be the effect.
The future research focuses are as follows

1.At present, $\mathrm{M} / \mathrm{g} / \mathrm{K}$ or $\mathrm{M} / \mathrm{g} / \mathrm{1}$ models are widely used in queuing models, but the arrival of vehicles may not conform to Poisson distribution in Chi square test, so it is impossible to apply the model to calculate. Some models other than Poisson distribution should be expanded.

2.Although the application of double charging is less, it has great potential. In general, a duplex channel will set up two toll windows. If more than two toll windows are set up, there is a lack of research.

\section{Reference}

1. Qu Qinzhou, Yang Kaige. Study on Minimum Clear Distance between Tunnel Exit and Expressway Mainline Toll Station[J]. IOP Conference Series: Earth and Environmental Science, 2021,634(1).

2. Kausalya. K, Bhavadarini. S, Gayathri. A. Automated Toll Booth using Morphological Edge Detection Algorithm[J]. International Journal of Recent Technology and Engineering (IJRTE), 2019, 8(4).

3. Yiqian Huang, Liang Chen, Yanwen Xia, Xiuliang Qiu. Design of Expressway Toll Station Based on Neural Network and Traffic Flow[J]. American Journal of Operations Research,2018,8(3).

4. Haiyun Huang, Junping Zhang, Yonghe Li. Analysis and prediction of operating vehicle load effects on Highway bridges under the weight charge policy[J]. IOP Conference Series: Earth and Environmental Science,2018,153(5)

5. Yiqian Huang, Liang Chen, Yanwen Xia, Xiuliang Qiu. Design of Expressway Toll Station Based on Neural Network and Traffic Flow[J]. American Journal of Operations Research,2018,8(3).

6. Xingqiang Zhang, Yubing Xiong, and Longfei Zuo."Research on Simulation and Optimization of Traffic Efficiency at Expressway Toll Stations near Big Cities". Proceedings of the 2011 Second ETP/IITA Conference on Telecommunication and Information (TEIN 2011 V1). Ed.. Engineering Technology Press, 2011, 140-143. 